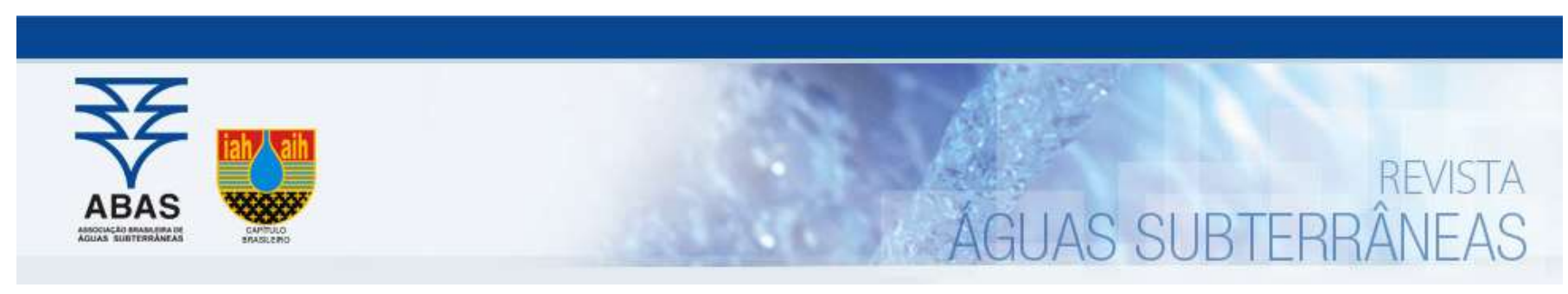

Artigos

\title{
Avaliação da distribuição de Gumbel na determinação de vazões mínimas da sub-bacia do Rio Negro
}

\section{Evaluation of the distribution of Gumbel in the determination of minimum flows of the Rio Negro sub-basin}

\author{
Gislaine Silva Pereira ${ }^{1}$; Fernanda Vilasboas Caldeira ${ }^{\boxplus}$ \\ 1 Universidade Estadual de Maringá (UEM), Maringá, PR, Brasil. \\ $\triangle$ gislainepereira-@hotmail.com, gislainepereira-@hotmail.com
}

Resumo

Palavras-chave:

Série histórica.

Mmodelo probabilístico.

Tempo de retorno.

\begin{abstract}
A água é o recurso natural que possibilita a vida na terra, e a falta de informações a respeito da gestão deste recurso dificulta o planejamento e a tomada de decisão adequados. Deste modo, em busca de determinação do risco de ocorrências de vazões mínimas, que podem vir a restringir o abastecimento de água, através de estudos de qualidade de água estimam-se a ocorrência de vazões utilizando curvas de probabilidades. Com isso, o objetivo do trabalho foi verificar a adequabilidade do modelo de probabilidade de Gumbel ao ajuste da série histórica de 33 anos de vazões mínimas provenientes da sub-bacia do Rio Negro. O Rio Negro abrange 22 municípios entre Santa Catarina e São Paulo possuindo várias estações fluviométricas em suas proximidades, na qual os dados escolhidos para este estudo foram da estação de fragosos (Águas do Paraná) no município de Piên, PR. A aplicação do modelo de Gumbel demonstrou que até o tempo de retorno de 20 anos há variabilidade nos dados, após isso a distribuição não se adequa a série histórica. Pode se concluir que a utilização do modelo de Gumbel não se adequou a todos os meses da série histórica em questão, comprovado com a realização de testes de aderência estatística.
\end{abstract}

Abstract

The water is a natural resource that enables life on earth and the lack of information regarding the management of this resource makes planning and decision making difficult. In order to determine the risk of occurrence of minimum flows, which may restrict the water supply, it is estimated by means of water quality studies the occurrence of flows using probability curves. Therefore, the objective of the study was to verify the suitability of the Gumbel probability model to fit the 33-year historical series of minimum flows from the Rio Negro sub-basin. The Rio Negro covers 22 cities between Santa Catarina and São Paulo with several fluviometric stations in its vicinity, in which the data chosen for this study were from the Water of Paraná station in Piên, PR. The application of the Gumbel model showed that until the return time of 20 years there is variability in the data, after which the distribution does not fit the historical series. The conclusion of the study is that the use of the Gumbel model did not fit all the months of the historical series in question, proven by the performance of statistical adherence tests.

DOI: http://dx.doi.org/10.14295/ras.v32i1.28926

\section{INTRODUÇÃO}

A água é um recurso natural indispensável para o desenvolvimento humano (OLIVEIRA e FIOREZE, 2011), e está disponível em fase sólida, líquida e gasosa (REICHARDT e TIMM, 2004). Diversas agências governamentais dão destaque a água como prioridade entre recursos naturais estratégicos (SILVA et al., 2006).

Segundo relatórios do Programa das Nações Unidas (PNUMA), os usos múltiplos da água, com base em seus volumes disponíveis, podem acarretar em crise no futuro, que vem a ser considerada sem precedentes de água na história da humanidade (LEME, 2003).

Silva (2003) afirma que o uso indiscriminado de água para fins de irrigação, levaria a uma situação crítica de conflitos entre demanda de água para consumo humano e para agricultura.

Deste modo, o acompanhamento da disponibilidade de água de uma bacia, auxilia na preservação da água, evitando assim, a escassez deste recurso no futuro, o que vem a auxiliar na identificação de cenários críticos devido a alta demanda, necessitando de orientação para utilização dos recursos hídricos (CBHLN, 2014).

Lopes et al. (2016) afirmam que a falta de informações a respeito da gestão de recursos hídricos é um dos principais fatores que dificultam o planejamento e a tomada de decisão adequada. 
Para gerir os recursos hídricos de forma adequada, é necessário que se tenha conhecimento da disponibilidade hídrica natural, que é caracterizada pelas vazões mínimas, se tornando fator essencial no planejamento e na gestão destes recursos (PRUSKI et al., 2015).

Para Tucci (2013), estas vazões mínimas são caracterizadas pelos menores valores de séries históricas anuais, sendo associadas a um tempo de duração.

Segundo o CBHLN (Comitê de Bacias Hidrográficas do Litoral Norte), deve-se adotar uma vazão mínima de referência, devido a previsão de vazão em sistema hídrico ser uma das técnicas utilizadas na diminuição do impacto das incertezas do clima sobre o gerenciamento de recursos hídricos (SOUZA et al., 2012).

As vazões mínimas mostram ser mais susceptíveis as ações antrópicas (PRUSKI et al., 2011), e desta maneira Tucci (2013) observou que os riscos de ocorrência de vazões mínimas, podem ser estimados utilizando curvas de probabilidade, pois estas vazões são necessárias em estudos de qualidade de água, regularização de vazões, abastecimento de água e irrigação.

Segundo Oliveira e Fioreze (2011), com o aumento da periodicidade de eventos extremos, a magnitude e frequência das vazões máximas aumentarão e as vazões mínimas irão diminuir em muitas regiões, resultando em conflitos de demandas e ofertas de água doce em escala mundial.

Sendo assim, é importante a realização de uma gestão considerada eficiente na utilização dos recursos hídricos, em que o uso de distribuições estatísticas para mensuração de eventos extremos de vazão, se tornam ferramentas imprescindíveis para esta gestão (SOUZA et al., 2012; ELESBON et al., 2015). Com isso, o objetivo do presente trabalho é a verificação da adequabilidade da distribuição de probabilidade de Gumbel para ajuste de séries históricas de vazões mínimas, para a sub-bacia hidrográfica do Rio Negro no Paraná.

\section{MATERIAIS E MÉTODOS}

A área de estudo está localizada na sub-bacia do Rio Negro em Piên (Latitude $26^{\circ}$ 05' 53" S, Longitude 49²5' 45” W) na região sul do Brasil, no sudoeste do Paraná. A classificação climática segundo Koppen é subtropical Cfb, que consiste em clima temperado com verão ameno, chuvas uniformemente distribuídas, com ausência de estação seca e precipitação média anual em torno de $1.500 \mathrm{~mm}$.

Para realização do estudo, foram coletados dados de vazões mínimas diárias para cada mês registrado pela estação fluviométrica de Águas do Paraná (6509000), fornecidos pela Agência Nacional de Águas (ANA). Os dados coletados foram totalizados em 37 anos, com 12 meses completos em cada ano, durante o período de 1968 a 2004. Os anos restantes que complementam a série histórica foram retirados por terem falhas, e apresentarem séries incompletas ou inconsistentes. Para a determinação das vazões mínimas da série histórica, foi utilizado o modelo de distribuição de Gumbel (WATANABE, 2013).

A distribuição de Gumbel para valores mínimos consiste em representar a parte inferior de uma determinada distribuição de dados e trata dos valores mínimos menos frequentes. A função acumulativa da probabilidade é dada pela relação $P(X \geq x)=e^{-\mathrm{e} \alpha(x-\beta)}, \quad$ em que $\quad \alpha_{\text {est }}=1,2826 / \sigma \quad \mathrm{e}$ $\beta_{\text {est }}=\bar{x}+0,451 \sigma$, onde $\sigma$ é definido como desvio padrão da série histórica; $\bar{x}$ é a média aritmética dos dados; $\beta$ e $\alpha$ são constantes de ajuste estimadas (WATANABE, 2013).

Para determinação dos valores de vazão mínima em função do tempo de retorno, será utilizada a relação de $x=\beta_{\text {est }}+\left\{\left(\frac{1}{\alpha_{\text {est }}}\right) * \ln \left[-\ln \left(1-\frac{1}{T R}\right)\right]\right\}$, onde $x$ corresponde a vazão mínima e TR o tempo de retorno em anos.

\subsection{Testes de aderência estatística}

Os testes que serão utilizados para verificar a aderência dos dados à distribuição de Gumbel são (referência): o coeficiente de ajuste (CA); coeficiente de massa residual (CRM); coeficiente de determinação $\left(R^{2}\right)$ e o teste de Kolmogorov-Smirnov.

O coeficiente de ajuste é obtido através da razão entre a dispersão dos valores observados e os calculados teoricamente, e deverá tender a um. 0 CA é encontrado pela relação $C A=\frac{\sum(M i+M x)^{2}}{\sum(T i-M x)^{2}}$, onde Mx é a média dos valores calculados pelo método; Mi é considerado o valor calculado pelo modelo e Ti são os valores observados pela série histórica.

O coeficiente de massa residual, consiste em um teste de aderência estatística utilizado para verificar se o modelo probabilístico se adequa a série. Segundo Aragão et al. (2012), os valores de CRM podem indicar superestimação, se CRM for maior que zero (CRM>0), e subestimação se CRM for menor que zero $(\mathrm{CRM}<0)$. Para valores de CRM igual a zero, indica-se uma condição ótima dos valores que são estimados pela distribuição de Gumbel.

O CRM é dado pela relação $C R M=\frac{\sum M i-\sum T i}{\sum M i}$, em que que Mi é considerado o valor calculado pelo modelo; Ti são os valores observados pela série histórica.

O coeficiente de determinação $\mathrm{R}^{2}$ é uma medida descritiva da qualidade do ajuste obtido. $0 \mathrm{R}^{2}$ pode variar de 0 a 1 , indicando em percentagem, o quanto o modelo consegue explicar os valores observados. Quanto maior o valor de $\mathrm{R}^{2}$, mais explicativo é o modelo.

O Teste de Kolmogorov-Smirnov (LILLIEFORS, 1967) é usado para determinar se duas distribuições de probabilidade subjacentes diferem uma da outra ou se a distribuição de probabilidade estimada se difere da probabilidade observada. Desta maneira, a obtenção de dmáx do teste de Kolmogorov-Smirnov (KS), se dá pela relação dmáx = máx $|F(x)-F o(x)|$, em que dmáx é a diferença máxima entre os valores da distribuição teórica e empírica; $F(x)$ é a frequência da amostra e $F o(x)$ é a frequência esperada. 


\section{RESULTADOS E DISCUSSÃO}

Com o aumento do tempo de retorno, ocorre a diminuição das vazões mínimas para todos os meses avaliados da série histórica (Tabela 1). As menores vazões mínimas da série em questão, foram encontradas para os meses de julho e setembro, para o tempo de retorno de 20 anos.

A medida em que ocorre o aumento do tempo de retorno em anos, as vazões mínimas tendem a diminuir, devido a diminuição da probabilidade na qual o valor será menor, para as vazões mínimas. As probabilidades observadas e estimadas de vazões mí- nimas para a série histórica estão representadas em Figura 1 e 2.

Observa-se pelo comportamento das curvas em todos os meses, que o modelo probabilístico não se adequou totalmente a série histórica, o que pode ser visto no comportamento das curvas de probabilidade estimada e observada.

Para o primeiro semestre avaliado da série histórica, os meses que melhor se ajustaram a distribuição de Gumbel, foram os meses de março e abril $\left(R^{2}=0,96\right.$ em ambos).

\begin{tabular}{ccccccc}
\multicolumn{6}{c}{ Tabela 1 - Vazões mínimas da sub-bacia do Rio Negro no Paraná em função do tempo de retorno em ano } \\
\hline \multirow{2}{*}{ TR (anos) } & Janeiro & Fevereiro & Março & Abril & Maio & Junho \\
\hline 2 & 14,1 & 15,3 & 13,8 & 11,5 & 11,5 & 11,3 \\
5 & 8,1 & 9,6 & 9,5 & 8,3 & 8,3 & 6,7 \\
10 & 4,1 & 5,8 & 6,7 & 6,3 & 6,3 & 3,6 \\
15 & 1,9 & 3,6 & 5,1 & 5,1 & 5,1 & 1,9 \\
20 & 0,3 & 2,1 & 4,0 & 4,3 & 4,3 & 0,7 \\
& Julho & Agosto & Setembro & Outubro & Novembro & Dezembro \\
2 & 12,2 & 13,8 & 11,5 & 13,2 & 13,2 & 11,1 \\
5 & 6,7 & 9,5 & 6,4 & 7,8 & 7,8 & 7,6 \\
10 & 3,1 & 6,7 & 3,0 & 4,3 & 4,3 & 5,3 \\
15 & 1,1 & 5,1 & 1,1 & 2,2 & 2,2 & 4,0 \\
20 & 0,0 & 4,0 & 0,0 & 0,8 & 0,8 & 3,1 \\
\hline
\end{tabular}

Os meses de dezembro, novembro e agosto (Figura 2), foram os meses que tiveram melhores ajustes com o modelo probabilístico utilizado $\left(R^{2}=0,95 ; 0,91\right.$ e 0,91 , respectivamente).

Haktanir (1991), ao estudar a adequabilidade de modelos probabilísticos na avaliação de vazões máximas anuais nos rios da Turquia, constatou que a distribuição de Gumbel não obteve melhor ajuste dos dados quando comparado as demais distribuições avaliadas, corroborando com a série histórica da sub-bacia do Rio Negro para vazões mínimas.
Leme (2003), utilizando a distribuição de Gumbel para estudo de vazão mínima do rio Jaguarim Mirim, constatou que poderiam ocorrer problemas com a gestão no uso de água da bacia, mostrando a adequabilidade da série de seu estudo com a distribuição de probabilidade, o que não foi notado no presente estudo.

A não adequabilidade do modelo de Gumbel e série histórica da sub-bacia do Rio Negro pode ter ocorrido por menor quantidade de dados (Série histórica de 37 anos), comparado ao estudo de Leme (2003) com dados de 50 anos. 
Figura 1 - Comportamento das vazões mínimas em relação às probabilidades observadas e estimadas de janeiro a junho (1968-2004) da sub-bacia do Rio Negro em Piên,PR
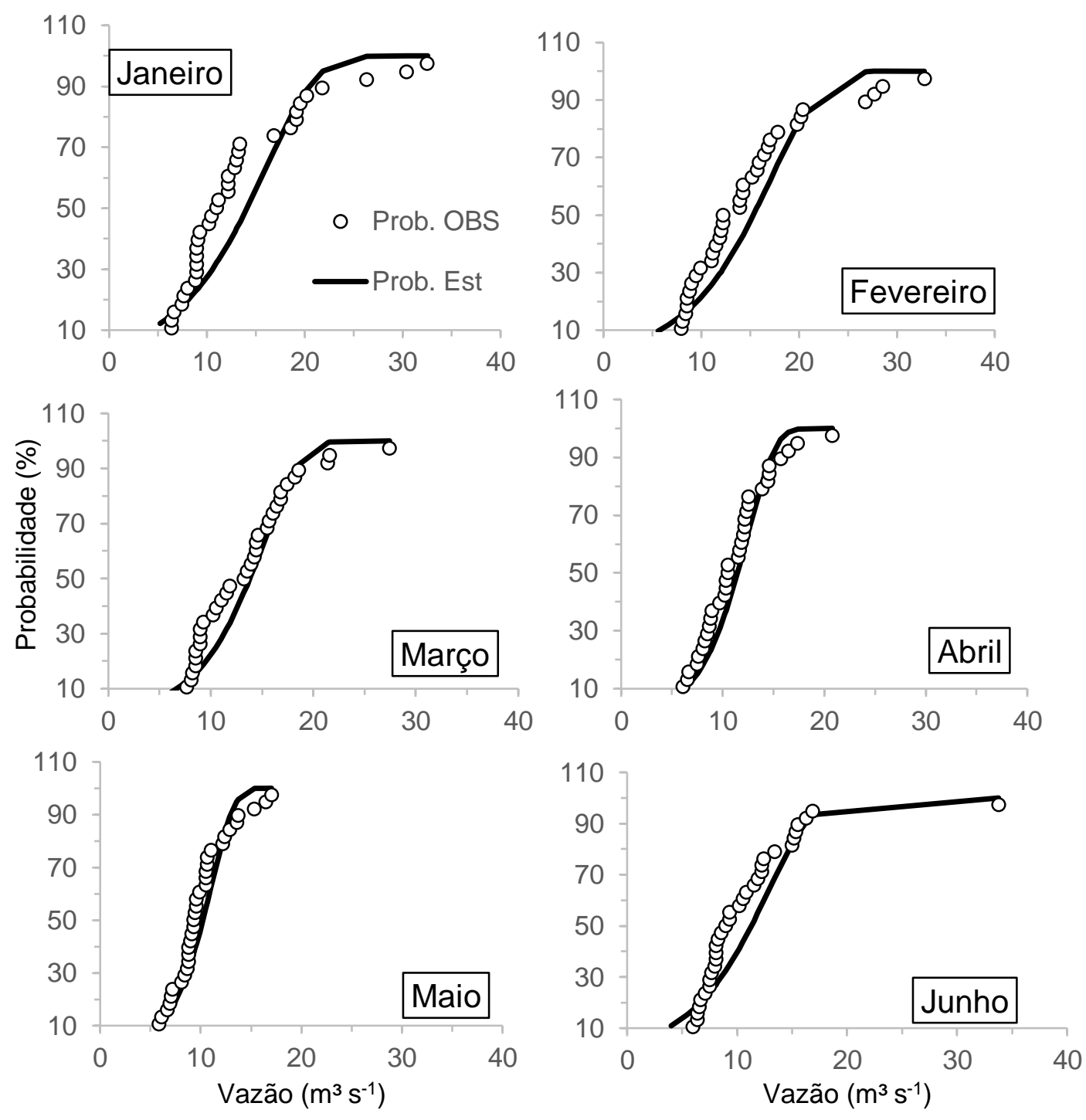
Figura 2 - Comportamento das vazões mínimas em relação às probabilidades observadas e estimadas de julho a dezembro (1968-2004) da sub-bacia do Rio Negro em Piên, PR
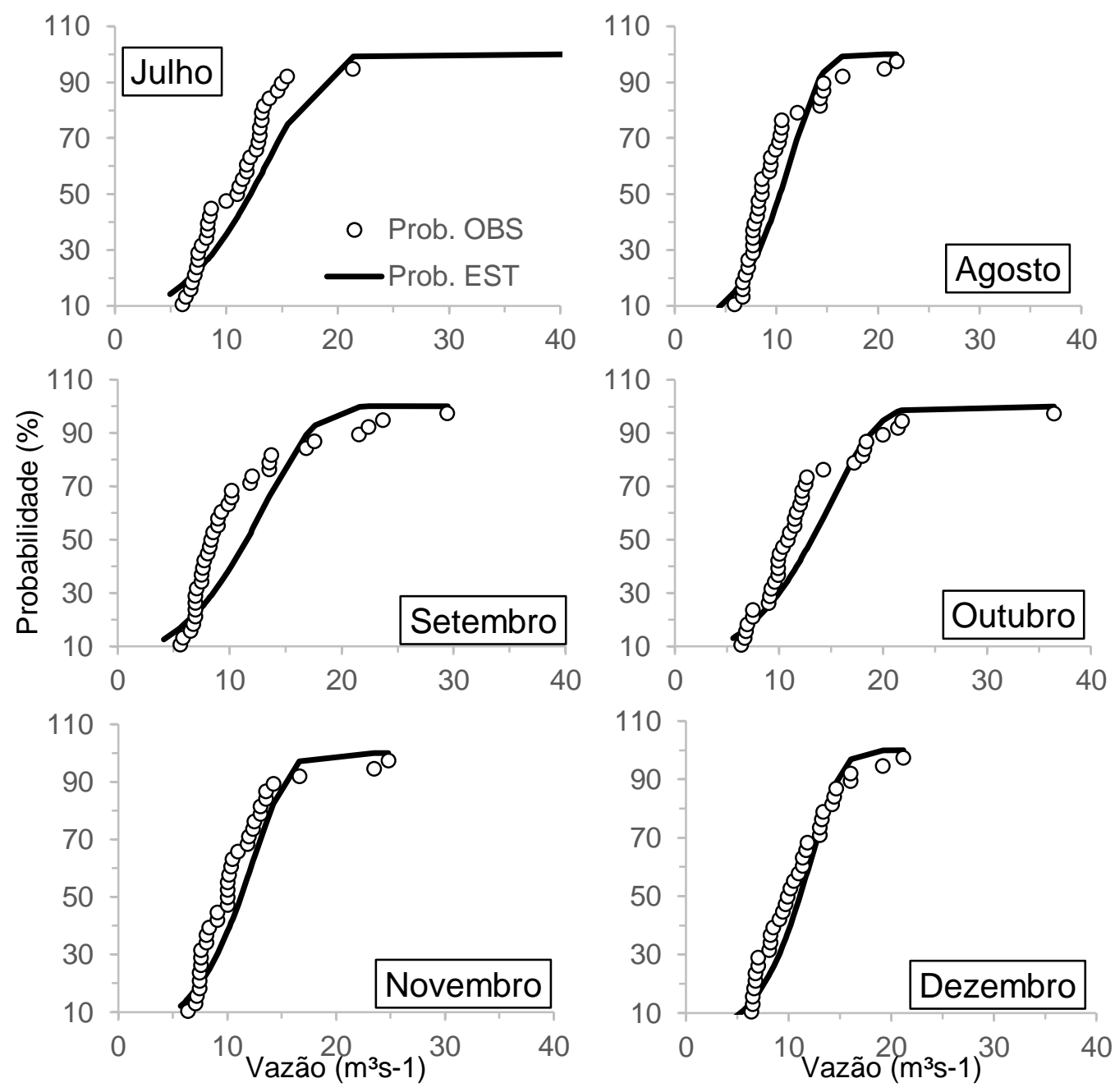

Souza et al. (2012), ao caracterizarem vazões mínimas de uma pequena bacia hidrográfica do Distrito Federal, puderam observar que a distribuição de Gumbel não representou bem os dados de vazões da série, o que foi visto no presente estudo.

A Tabela 2 apresenta os valores obtidos com a realização de testes de aderência estatística para a verificação de adequabilidade da distribuição de Gumbel com os dados de vazões mínimas para a sub-bacia do Rio Negro.

O mês de março foi o mês que melhor se ajustou para todos os testes de aderência empregados, então se pode afirmar que a distribuição de Gumbel se adequou aos dados para o mês em questão.

Os maiores coeficientes de ajuste (CA), foram encontrados nos meses de fevereiro, março e dezembro. 0 teste de aderência $C A$ não se adequou bem a distribuição, pois nenhum dos meses obtiveram valores que tenderam a um.

Em relação ao coeficiente de massa residual (CRM) os valores para todos os meses foram subestimados ao se utilizar a distribuição de Gumbel, o que não corrobora com Lopes et al. (2016), estudando vazões máximas e mínimas para a bacia do Rio Ivaí,

estes afirmaram que a distribuição Gumbel se ajustou adequadamente para o teste de aderência de coeficiente de massa residual.

Aragão (2012), constatou em seu estudo sobre chuvas diárias, que o valores de $R^{2}$ permaneceram constantes $\left(R^{2}=0,99\right)$, o que não foi observado para a série histórica em questão, em que houve variações do coeficiente de determinação. Os meses que obtiveram melhor ajuste pelo coeficiente de determinação $\left(R^{2}\right)$ foram os meses de março e abril.

$O$ valor crítico encontrado para o teste K-S foi de $\alpha=0,22$. Observa-se que de todos os meses avaliados, a série não se adequou aos dados apenas para os meses de janeiro, agosto, setembro e outubro, ou seja, os dados não se adequaram normalmente a distribuição de Gumbel. É necessário que se realize a correção dos resíduos para posterior avaliação de normalidade dos dados para os meses não ajustados.

Finkler et al. (2015) ao avaliarem funções de distribuição de probabilidade na determinação de vazão mínima para a bacia hidrográfica do Arroio Belo, constataram que o teste de K-S indicou completa restrição a distribuição de Gumbel em todos os meses analisados, o que não ocorreu no presente estudo. 
Tabela 2 - Testes de Aderência Estatística para a distribuição de Gumbel de dados históricos de vazões mínimas da sub-bacia do Rio Negro em Piên, PR

\begin{tabular}{lcccc}
\hline \multicolumn{1}{c}{ Mês } & CA & CRM & $\begin{array}{c}\text { Testes de Aderência } \\
\mathbf{R}^{2}\end{array}$ & K-S \\
\hline Janeiro & 0,83 & $-0,21$ & 0,86 & 0,25 \\
Fevereiro & 1,24 & 0,19 & 0,91 & 0,19 \\
Março & 1,08 & 0,08 & 0,96 & 0,15 \\
Abril & 0,77 & $-0,30$ & 0,96 & 0,14 \\
Maio & 0,88 & $-0,14$ & 0,94 & 0,17 \\
Junho & 0,74 & $-0,34$ & 0,90 & 0,21 \\
Julho & 0,63 & $-0,58$ & 0,91 & 0,23 \\
Agosto & 0,79 & $-0,27$ & 0,84 & 0,25 \\
Setembro & 0,75 & $-0,33$ & 0,81 & 0,28 \\
Outubro & 0,78 & $-0,29$ & 0,85 & 0,27 \\
Novembro & 0,73 & $-0,37$ & 0,91 & 0,21 \\
Dezembro & 1,28 & 0,22 & 0,95 & 0,14 \\
\hline
\end{tabular}

\section{CONCLUSÃO}

A distribuição de Gumbel não foi adequada para a série de dados da sub-bacia do Rio Negro, o que pode ser visto com os testes de aderência estatística. 0 mês de março melhor se adequou a distribuição de probabilidade.

\section{REFERÊNCIAS}

AGENCIA NACIONAL DE ÁGUAS - ANA. Disponivel em:< http://www2.ana.gov.br/Paginas/default.aspx>. Acesso em: 20 de Agosto de 2017.

ARAGÃO, R., SANTANA, G.R., COSTA, C.E, CRUZ, M.A., FIGUEIREDO, E.F., SRINIVASAM, V. Chuvas Intensas para o estado de Sergipe com base em dados desagregados de chuva diária. Revista Brasileira de Engenharia Agrícola e Ambiental, v 17, n. 3, pg.243-252, 2012.

COMITÊ DE BACIAS HIDROGRÁFICAS DO LITORAL NORTE - CHB. Relatório técnico CBH-LN disponibilidade hídrica das bacias hidrográficas do litoral norte (UGRHI 03). São Paulo, 2014. Disponivel em:< http://www.sigrh.sp.gov.br/public/uploads/documents/8163/relatorio-tecnico-cbh-Incriticidade-2014.pdf>. Acesso em 20 de Agosto de 2017.

ELESBON, A.A.A.; da SILVA, D.D.; SEDIYAMA, G.C.; GUEDES, H.A.S.; RIBEIRO, C.A.A.; RIBEIRO, C.B. Multivariate statistical analysis to support the minimum streamflow regionalization. Engenharia Agrícola, v. 35, n.5, 2015.

FINKLER, N.R.; MENDES, L.A.; SCHENEIDER, E.H.M.; BORTOLIN, T.A.; SCHENEIDER, V.E. Comparação de funções de distribuição de probabilidades na determinação de vazão mínima anual e sazonal. Scientia Cum Industria, v.3, n.2, p.42-49, 2015.

HAKTANIR, T. Statistical modellinf of annual maximum flows in Turkish rivers. Hydrological Sciences Journal, v.36, n.4, 1991.

LEME, E.J. Hidrologia Estatística da vazão mínima do Rio Jaguari Mirim. Revista Ecossistema, v. 27, n.2, jan-dez 2003.

LILLIEFORS, H.W. On the Kolmogorov-Smirnov test for normality with mean and variance unknown. Journal of the American Statistical Association, v. 62, 1967.
LOPES, T.R.; PRADO, G.; ZOLIN, C.A.; ANTONIEL, L.S. Regionalização de vazões máximas e mínimas para a bacia do Rio Ivaí - PR. Revista Irriga, Botucatu, PR, v.21, n.1, p. 188- 201, 2016.

OLIVEIRA, L.F.C.; FIOREZE, A.P. Estimativas de vazões mínimas mediante dados pluviométricos na Bacia Hidrográfica do Ribeirão Santa Bárbara, Goiás. Revista Brasileira de Engenharia Agrícola e Ambiental, v.15, n.1, 2011.

OLIVEIRA, L.F., FIOREZE, A.P. Estimativas de vazões mínimas mediante dados pluviométricos na Bacia Hidrográfica do Ribeirão Santa Bárbara, Goiás. Revista Brasileira de Engenharia Agrícola e Ambiental, v.15, n.1, pg.9-15, 2011.

PRUSKI, F.F.; RODRIGUEZ, R.G.; SOUZA, J.F.; SILVA, B.M.B.; SARAIVA, I.S. Conhecimento da disponibilidade hídrica natural para a gestão de recursos hídricos. Engenharia Agrícola, v.31, n.1, 2011.

PRUSKI, F.F.; RODRIGUEZ, R.G.; NUNES, A.A.; PRUSKI, P.L.; SINGH, V.P. Low-flow estimates in regions of extrapolation of the regionalization equations: a new concept. Engenharia Agrícola, v.35, n.5, 2015.

REICHARDT K., TIMM, L.C. Solo, planta e atmosfera: conceitos, processos e aplicações. 1. ed. 2004.

SILVA, A.M., OLIVEIRA, P.M., MELLO, C.R., PIERANGELI, C. Vazões mínimas e de referência para outorga na região do Alto Rio Grande, Minas Gerais. Revista Brasileira de Engenharia Agrícola e Ambiental, v.10, n.2, pg.374-380, 2006.

SILVA, C.L. Análise estatística das características de vazão do córrego Capetinga. Revista Brasileira de Engenharia Agrícola e Ambiental, v.7, n.2, p.311-317. 2003.

SOUZA, F.A.O.; SILVA, C.L.; MAGGIOTTO, S.R.; OLIVEIRA JUNIOR, M.P.O. Caracterização das vazões em uma pequena bacia hidrográfica do Distrito Federal, Brasil. Revista Brasileira de Engenharia Agrícola e Ambiental, v.16, n.1, 1012.

TUCCI, C. E. Hidrologia: ciência e aplicação. 4. ed. Porto Alegre: Editora da UFRGS/ABRH, 2013.

WATANABE, F.M. Análise do método de Gumbel para cálculo de vazões de dimensionamento de vertedouros. Monografia (Graduação em Engenharia Elétrica) - Escola de Engenharia de São Carlos, USP, 2013. 